\title{
A Randomized Controlled Trial of Motavizumab Versus Palivizumab for the Prophylaxis of Serious Respiratory Syncytial Virus Disease in Children With Hemodynamically Significant Congenital Heart Disease
}

\author{
TIMOTHY F. FELTES, HENRY M. SONDHEIMER, ROBERT M. R. TULLOH, BRIAN S. HARRIS, KATHRYN M. JENSEN, \\ GENEVIEVE A. LOSONSKY, AND M. PAMELA GRIFFIN; MOTAVIZUMAB CARDIAC STUDY GROUP
}

\begin{abstract}
Department of Pediatrics [T.F.F.], The Ohio State University and Nationwide Children's Hospital, Columbus, Ohio 43205; The Association of American Medical Colleges [H.M.S.], Washington, DC 20037; Bristol Congenital Heart Centre [R.M.R.T.], Bristol Royal Hospital for Children, Bristol BS2 8BJ, United Kingdom; Departments of Biostatistics [B.S.H., K.M.J.] and Clinical Development [G.A.L., M.P.G.], MedImmune, LLC, Gaithersburg, Maryland 20878
\end{abstract}

\begin{abstract}
Children with hemodynamically significant congenital heart disease (CHD) are at risk for serious respiratory syncytial virus (RSV) disease. This study was designed to assess the safety and tolerability of motavizumab versus palivizumab in children with CHD and was not powered for efficacy. Patients $(n=1236)$ aged $\leq 24$ mo were randomized to receive five monthly doses $(15 \mathrm{mg} / \mathrm{kg})$ of motavizumab or palivizumab during the RSV season. Adverse events (AEs) and serious AEs (SAEs) were recorded through $30 \mathrm{~d}$ after the last dose. RSV hospitalizations and RSV outpatient medically attended lower respiratory tract infections (MALRI; season 2) were summarized. Approximately 93 and $50 \%$ of patients reported an AE or SAE, respectively. Skin events occurred in $19.3 \%$ of motavizumab recipients and $16.2 \%$ of palivizumab recipients. Rates of hospitalizations and RSV MALRI were similar between treatment groups [relative risk (RR): 0.75; 95\% CI, 0.34-1.59 and RR: 0.49; 95\% CI, 0.10-1.99, respectively; both $p>0.05$ ]. Motavizumab and palivizumab had similar safety profiles in children with hemodynamically significantly CHD; with the exception of skin events which were increased in motavizumab recipients. Safety and efficacy were consistent with another study comparing motavizumab with palivizumab in premature infants without CHD. (Pediatr Res 70: 186-191, 2011)
\end{abstract}

$\mathrm{T}$ he World Health Organization estimates the annual global burden of respiratory syncytial virus (RSV) infection to be 64 million cases (1). Children at high risk for severe RSV disease [premature infants, infants with chronic lung disease (CLD) of prematurity, and infants with congenital heart dis-

Received November 10, 2010; accepted February 10, 2011.

Correspondence: Timothy F. Feltes, M.D., Nationwide Children's Hospital, 700 Children's Drive, ED617, Columbus, OH 43205; e-mail: timothy.feltes@nationwidechildrens.org

This study was sponsored by MedImmune, LLC. MedImmune was involved in the study design; collection, analysis, and interpretation of data; the writing of this manuscript; and the decision to submit the manuscript for publication. T.F.F. is a consultant for MedImmune and a former member of MedImmune's speakers bureau. H.M.S. is a former consultant and former member of the MedImmune speakers bureau through 2007. The Bristol Royal Hospital for Children received investigator fees for the study of motavizumab, but R.M.R.T. did not personally receive any financial remuneration directly or indirectly for participating in the project. T.F.F., H.M.S., or R.M.R.T. have no financial interest in MedImmune or related companies. B.S.H., K.M.J., G.A.L., and M.P.G. are employees of MedImmune.

Supplemental digital content is available for this article. Direct URL citations appear in the printed text and are provided in the HTML and PDF versions of this article on the journal's Web site (www.pedresearch.org). ease (CHD)] $(2,3)$ are more likely to require medical attention for RSV and are hospitalized 4-5 times more frequently than non-high-risk children (4-7).

Palivizumab (MedImmune, Gaithersburg, MD) is a humanized MAb that recognizes a highly conserved neutralizing epitope on the fusion protein of RSV (8) and is recommended for RSV prophylaxis of at-risk children $(9,10)$. Monthly prophylaxis with palivizumab reduced RSV hospitalizations by $\sim 50 \%$ compared with placebo in premature infants (11) and in children aged $\leq 24$ mo with hemodynamically significant CHD (12).

Motavizumab (MedImmune, Gaithersburg, MD) is an RSV-specific MAb with significantly higher in vitro binding affinity for RSV fusion protein than palivizumab $(13,14)$. In the cotton rat model, motavizumab reduced nasal and lung RSV titers to $1 / 25$ th and 1/100th, respectively, of the levels that were observed with palivizumab and showed $\sim 20$-fold improved activity in microneutralization studies $(14,15)$. The serum pharmacokinetic profile of motavizumab is consistent with that of palivizumab, with no dose-limiting toxicities $(16,17)$.

In an earlier study in high-risk preterm children, motavizumab was shown to be statistically noninferior to palivizumab in reducing RSV hospitalizations (the primary end point) and superior to palivizumab by reducing outpatient RSV medically attended lower respiratory tract infections (MALRI), a secondary end point (18).

The current study evaluated motavizumab compared with palivizumab for prophylaxis of RSV disease in children with hemodynamically significant CHD. The primary objective of this study was to describe the safety and tolerability of motavizumab compared with palivizumab. Rates of RSV hospitalization and RSV outpatient MALRIs were also examined.

\footnotetext{
Abbreviations: ADA, antidrug antibody; AE, adverse event; CHD, congenital heart disease; CLD, chronic lung disease; EU, European Union; ITT, intent-to-treat; MALRI, medically attended lower respiratory tract infection; ROW, rest of world; RR, relative risk; RSV, respiratory syncytial virus; SAE, serious adverse event
} 


\section{METHODS}

Study design. Because a larger phase 3 study has been conducted and powered to assess the safety and efficacy of motavizumab in a population of preterm infants at risk for RSV illness (18), this phase 2, randomized, multinational, double-blind, palivizumab-controlled study conducted over two RSV seasons (2005-2006 and 2007-2008) was designed to evaluate the safety and tolerance of motavizumab in children with CHD [ClinicalTrials. gov identifiers: NCT00240890 (season 1) and NCT00538785 (season 2)]. Sample size calculations and expected hospitalization rates were based on data from previous studies, including the study by Carbonell-Estrany et al. (18). Because of the efficacy of the comparator palivizumab, the sample size needed to sufficiently power this study for noninferiority would have required $>6000$ subjects. To demonstrate superiority would have required an even larger sample size and studies of this magnitude could not be conducted in this patient population in a reasonable time period.

The study was conducted in accordance with the Declaration of Helsinki and the International Conference on Harmonisation Guidelines for Good Clinical Practice and was approved by the Institutional Review Board or Independent Ethics Committee of each participating center. Centers in the United States were also compliant with the Health Insurance Portability and Accountability Act of 1996. Parents or legal guardians provided written informed consent for each child. Patients were randomly assigned 1:1 (using an interactive voice-response system) to receive monthly intramuscular doses of $15 \mathrm{mg} / \mathrm{kg}$ motavizumab or palivizumab for a total of five doses. Randomization was stratified by site and the presence or absence of cyanotic CHD. Motavizumab and palivizumab were provided in identical vials in coded kits. All study personnel were blinded to treatment assignments. Each patient participated during a single RSV season.

Study end points. Safety and tolerability were assessed primarily by summarizing adverse events (AEs) and serious AEs (SAEs) reported through study day 150 ( $30 \mathrm{~d}$ after last dose). An AE was any change from the patient's baseline status. SAEs were events that resulted in a significant disability (a substantial impairment of baseline function) or death, required or prolonged hospitalization, or otherwise were considered an important medical event. Site investigators assessed the severity of AEs and SAEs [level 1 (mild), level 2 (moderate), level 3 (severe), and level 4 (life threatening)] and relationship to study drug (none, remote, possible, probable, and definite). Efficacy end points were the incidences of RSV hospitalization and RSV outpatient MALRI (for patients in season 2 only) through study day 150. RSV hospitalization was defined as hospitalization for cardiac/respiratory symptoms accompanied by a positive RSV test or a new onset of RSV-positive lower respiratory illness with worsening respiratory status while already in the hospital or death caused by RSV. RSV outpatient MALRI was an outpatient medically attended event diagnosed as a lower respiratory illness accompanied by a positive RSV test.

Participants. Patients were children aged $\leq 24$ mo at randomization with documented hemodynamically significant CHD defined as uncorrected or palliated cyanotic CHD or acyanotic CHD associated with documented pulmonary hypertension (systolic pulmonary arterial pressure $\geq 40 \mathrm{~mm} \mathrm{Hg}$ ) and/or a requirement for daily medication to manage congestive heart failure. These criteria intentionally excluded patients with uncomplicated or insignificant CHD and are consistent with those from a previous placebo-controlled study of palivizumab in high-risk children with CHD (12). Full exclusion criteria are available at supplemental methods, http://links.lww.com/PDR/ A76. Children with cyanotic CHD included those with the most commonly encountered cyanotic cardiac lesions (Table S1, http://links.lww.com/PDR/ A76). Children who did not have one of the designated diagnoses to be included in the cyanotic stratum were classified as "other" anticipating that this group would largely comprise children with acyanotic CHD lesions.

Laboratory assessments. Respiratory secretions (aspirates or posterior nasopharyngeal swabs, or tracheal secretions, if intubated) for RSV testing were collected within $2 \mathrm{~d}$ of or as soon as possible after a cardiac/respiratory hospital admission or nosocomial event, or an outpatient MALRI (season 2 only). At a central laboratory, personnel who were blind to treatment assignment tested respiratory secretions for RSV A and RSV B using validated real-time RT-PCR diagnostic assays, as described previously (18).

Serum samples for motavizumab concentrations and antidrug antibody (ADA) were obtained from all patients before the first, second, and fifth doses of study drug with an additional randomly assigned sample taken before the third or fourth dose of study drug. ADA for motavizumab and palivizumab was assessed using a drug-tolerant electrochemiluminescent assay at the last available postbaseline sample only (18). A positive antimotavizumab titer was defined as $\geq 1: 30$.

Study populations. The intent-to-treat (ITT) population included all randomized patients. The ITT outpatient MALRI population included all patients randomized during season 2 . The safety population included all patients who received any study medication and had any safety follow-up. Patients who were randomly assigned to motavizumab and received commercial palivizumab within 3 mo before receiving study drug were excluded from the safety, ADA, and pharmacokinetic analyses.

Statistical analyses. The sample size was sufficient to estimate the rates of AEs assuming rates similar to those observed in the phase 3 study of palivizumab in CHD patients (9). AEs and SAEs were summarized by system organ class and preferred term using the Medical Dictionary for Regulatory Activities, version 11.1, by severity and relationship to study drug, primary cardiac lesion, and presence or absence of cyanotic CHD. Comparisons between treatment groups were made for the total number of subjects reporting at least one $\mathrm{AE}$ and at the system organ class level using a 2 -sided $95 \%$ exact binomial $\mathrm{CI}$ around the observed rate difference and $p$ value for the observed rate difference (19). No adjustments were made for multiple comparisons.

Mean trough serum concentrations of motavizumab at each time point were summarized using descriptive statistics. The first analysis of mean trough serum concentrations of motavizumab excluded serum concentrations that were obtained from patients after cardiac surgery with cardiopulmonary bypass; a post hoc analysis included those concentrations that were obtained after cardiac surgery with cardiopulmonary bypass in patients who received an additional replacement dose of motavizumab. The number and percentages of patients with ADA to motavizumab or palivizumab were summarized.

As noted previously, the current study was not powered to demonstrate statistical superiority or noninferiority of motavizumab over palivizumab regarding efficacy. RSV hospitalization rates and RSV outpatient MALRI rates were evaluated using a descriptive summary of observed relative risk (RR); the corresponding 2-sided 95\% CI around the observed RR was determined using the exact conditional binomial method conditioning on the total number of cases with midprobability adjustment (20). Because patients were stratified by the presence of cyanotic CHD, the analysis included a 2-level categorical covariate to control for the effect. Because this study was not powered for statistical comparisons, the upper bound of the $95 \%$ CI around the observed RR was not compared with any prespecified noninferiority or superiority margins.

\section{RESULTS}

A total of 1236 patients (motavizumab, $n=624$; palivizumab, $n=612$ ) were randomized at 134 sites [North America, 51; European Union (EU), 56; rest of world (ROW), 27] between October 21, 2005, and December 14, 2005, in season 1 and at 100 sites (North America, 37; EU, 48; ROW, 15) between October 2, 2007, and December 31, 2007, in season 2. Data for one patient who was withdrawn from the study after being declared ineligible by the local ethics committee were excluded from all analysis populations (Figure S1, http:// links.lww.com/PDR/A76). Overall, 97.0 and $97.2 \%$ of motavizumab and palivizumab recipients, respectively, completed the study; 95.2 and $94.6 \%$ of patients, respectively, received all five monthly doses of study drug. Treatment groups were well matched with regard to demographic and baseline variables (Table 1). Overall, 665 of 1235 (53.8\%; motavizumab, 346; palivizumab, 319) patients were stratified as cyanotic and 570 (46.2\%; motavizumab, 277; palivizumab, 293) were stratified as "other" (Table S1, http://links.lww.com/ PDR/A76). The most common cardiac lesions in the cyanotic stratum were tetralogy of Fallot (16.3\%) and single ventricle (including hypoplastic left or right heart, 15.3\%). The most common cardiac lesions in the "other" stratum were ventricular septal defect and atrioventricular septal defect (23.0 and $7.9 \%$, respectively).

Safety and tolerability. More than $90 \%$ of all patients reported $\geq 1 \mathrm{AE}$ and $\sim 50 \%$ had $\geq 1 \mathrm{SAE}$ (Table 2). Most AEs were level 1 or 2 in severity, and the rates of level 3 and 4 AEs were similar between treatment groups (Table 2). The most commonly reported AEs were pyrexia (motavizumab, 29.8\%; 
Table 1. Demographic characteristics, RSV risk factors, and status of CHD of the ITT population at study entry

\begin{tabular}{|c|c|c|}
\hline Characteristic & $\begin{array}{l}\text { Motavizumab } \\
\quad(n=623)\end{array}$ & $\begin{array}{c}\text { Palivizumab } \\
(n=612)\end{array}$ \\
\hline \multicolumn{3}{|l|}{ Demographic characteristics } \\
\hline Age, mean (SD), mo & $8.47(6.40)$ & $8.18(6.51)$ \\
\hline GA at birth, mean (SD), wk & $38.5(2.1)$ & $38.5(2.0)$ \\
\hline Boys, $n(\%)$ & $341(54.7)$ & $314(51.3)$ \\
\hline \multicolumn{3}{|l|}{ Race/ethnicity, $n(\%)$} \\
\hline White/non-Hispanic & $540(86.7)$ & $529(86.4)$ \\
\hline Black & $23(3.7)$ & $20(3.3)$ \\
\hline Hispanic & $21(3.4)$ & $23(3.8)$ \\
\hline Asian & $10(1.6)$ & $8(1.3)$ \\
\hline Other & $29(4.7)$ & $32(5.2)$ \\
\hline \multicolumn{3}{|l|}{ Geographic region } \\
\hline North America* & $182(29.2)$ & $168(27.5)$ \\
\hline $\mathrm{EU} \dagger$ & $262(42.1)$ & $261(42.6)$ \\
\hline Rest of world $\ddagger$ & $179(28.7)$ & $183(29.9)$ \\
\hline Multiple births (yes), $n(\%)$ & $32(5.1)$ & $22(3.6)$ \\
\hline Weight at birth, mean (SD), kg & $3.08(0.65)$ & $3.07(0.63)$ \\
\hline Weight at study d 0 , mean (SD), $\mathrm{kg}$ & $6.81(2.61)$ & $6.63(2.60)$ \\
\hline \multicolumn{3}{|l|}{ RSV risk factors } \\
\hline $\begin{array}{l}\text { Number of adults in household, mean } \\
\text { (SD) }\end{array}$ & $2.4(1.0)$ & $2.3(0.9)$ \\
\hline $\begin{array}{l}\text { Children younger than } 6 \mathrm{y} \text { in household, } \\
\qquad(\%)\end{array}$ & $309(49.6)$ & $272(44.4)$ \\
\hline Family history of atopy, $n(\%) \S$ & $242(38.8)$ & $232(37.9)$ \\
\hline$\geq 1$ smoker in household, $n(\%)$ & $237(38.0)$ & $234(38.2)$ \\
\hline \multicolumn{3}{|l|}{ CHD status } \\
\hline Cyanotic stratum & $346(55.5)$ & $319(52.1)$ \\
\hline $\begin{array}{l}\text { Previous cardiac surgery or interventional } \\
\text { catheterization, } n(\%)\end{array}$ & $344(55.2)$ & $306(50.0)$ \\
\hline Previous diagnostic catheterization, $n(\%)$ & $204(32.7)$ & $174(28.4)$ \\
\hline Pulmonary hypertension, $n(\%) \|$ & $137(22.0)$ & $150(24.5)$ \\
\hline Increased pulmonary blood flow, $n(\%)$ & $227(36.4)$ & $237(38.7)$ \\
\hline \multicolumn{3}{|l|}{ Level of cardiac failure, $n(\%)$} \\
\hline Controlled with medication & $386(62.0)$ & $376(61.4)$ \\
\hline Uncontrolled with medication & $8(1.3)$ & $20(3.3)$ \\
\hline
\end{tabular}

* Canada and the United Sates.

$\dagger$ Austria, Belgium, Czech Republic, France, Germany, Hungary, Poland, Spain, Sweden, and the United Kingdom.

¥ Bulgaria, Israel, Lebanon, and Russia.

$\S$ Atopy was defined as asthma, hay fever, or eczema.

|| Moderate or severe.

Table 2. Overview of safety data (safety population)

\begin{tabular}{lccc}
\hline & Motavizumab & Palivizumab & \\
\multicolumn{1}{c}{ Outcome, $n(\%)$} & $575(93.0)$ & $566(92.5)$ & 0.742 \\
$\geq 1 \mathrm{AE}$ & $51(8.3)$ & $54(8.8)$ & 0.760 \\
$\geq 1$ related $\dagger \mathrm{AE}$ & $184(29.8)$ & $203(33.2)$ & 0.219 \\
$\geq 1$ level $3 \mathrm{AE}$ & $64(10.4)$ & $68(11.1)$ & 0.713 \\
$\geq 1$ level 4 AE & $292(47.2)$ & $304(49.7)$ & 0.424 \\
$\geq 1$ SAE & $5(0.8)$ & $6(1.0)$ & 0.772 \\
$\geq 1$ related $\dagger$ SAE & $0(0.0)$ & $1(0.2)$ & 0.498 \\
$\geq 1$ AE resulting in discontinuation & & & \\
$\quad$ of study drug & $0(0.0)$ & $1(0.2)$ & 0.498 \\
$\geq 1$ related $\dagger$ AE resulting in & & & \\
$\quad$ discontinuation of study drug & $9(1.5)$ & $10(1.6)$ & 0.822 \\
Deaths, $n(\%)$ & &
\end{tabular}

$* p$ values were calculated using Fisher exact test.

$\dagger$ Related is defined as possibly, probably, or definitely related to study drug.

palivizumab, 29.2\%), upper respiratory tract infection (27.3 versus $28.1 \%$ ), cough (14.9 versus $11.6 \%$ ), rhinitis (14.7 versus $12.6 \%$ ), and otitis media (12.5 versus $11.4 \%$; Table S2, http://links.lww.com/PDR/A76). The AE profiles for both treatment groups were similar, with only six events differing by $\geq 2 \%$ points between groups: cough (motavizumab, $14.9 \%$; palivizumab, $11.6 \%)$, rhinitis (14.7 versus $12.6 \%)$, constipation ( 7.1 versus $5.1 \%)$, tetralogy of Fallot (6.1 versus $8.2 \%)$, irritability (3.6 versus $6.2 \%$ ), and alanine aminotransferase increased (2.1 versus $4.2 \%$ ). The proportion of patients with AEs considered by the site investigator to be related to study medication was similar between treatment groups. Among motavizumab recipients, 76 AEs were considered to be related to treatment in 51 patients (8.3\%); 69 AEs were reported to be treatment related in $54(8.8 \%)$ palivizumab recipients (Table 2).

The body systems with the largest percentage-point difference between treatment groups were congenital, familial, and genetic disorders (motavizumab, 28.3\%; palivizumab, 34.3\%) and skin and s.c. tissue disorders (motavizumab, 19.3\%; palivizumab, 16.2\%). Additional analyses conducted to evaluate the rate difference between the two treatment groups found in congenital, familial, and genetic disorders showed that the difference mostly was because of an increase in AEs associated with unplanned cardiac/respiratory hospitalizations in palivizumab patients compared with motavizumab patients [motavizumab, 154 (24.9\%); palivizumab, 180 (29.4\%)]. There was a small increase in AEs associated with planned cardiac/respiratory hospitalizations in palivizumab patients compared with motavizumab patients [motavizumab, 9 (1.5\%); palivizumab, 17 (2.8\%)].

Skin events. From the skin and s.c. tissue disorder body system, a list of preferred terms was created and reviewed for appropriateness to the patient population. Additional terms considered to be indicative of a possible cutaneous manifestation (i.e. hypersensitivity reaction) were added. This list of preferred terms for skin events considered "of interest" was then subcategorized into "nonspecific" event terms such as rash and more "specific" event terms potentially consistent with hypersensitivity such as urticaria (Table S3, http://links. lww.com/PDR/A76). Skin events were further analyzed for occurrence relative to study drug dosing and the level of severity (Figure S2, http://links.lww.com/PDR/A76). By this analysis, the overall rate of skin events of interest was $10.2 \%$ in motavizumab recipients and $7.2 \%$ in palivizumab recipients. Most skin events were nonspecific rashes that were mild, transient, and did not result in discontinuation of study drug. The only dosing discontinuation in the study was in a palivizumab recipient who experienced an event of a maculopapular rash. More motavizumab recipients experienced events consistent with possible cutaneous hypersensitivity (including urticaria and drug hypersensitivity) within $2 \mathrm{~d}$ of dosing [motavizumab, 6 of 618 (1.0\%); palivizumab, 1 of 612 $(0.2 \%)]$. Among these six specific skin events in motavizumab recipients that occurred within $2 \mathrm{~d}$ of dosing, four occurred after the fifth dose, and two children were redosed without a recurrence of the skin event. Four of the six motavizumab recipients with a specific skin event that occurred within $2 \mathrm{~d}$ of dosing received steroids or antihistamines. One motavizumab recipient experienced a serious level 3 event of urticaria $\sim 15$ min after receipt of the fifth dose of motavizumab; the patient experienced generalized urticaria on his head, upper and lower trunk, extremities, and swollen eyelids. The patient was 
treated with steroids and an antihistamine and was admitted to the hospital for observation; however, his symptoms resolved within $6 \mathrm{~h}$ and he was discharged.

Skin events that were reported as level 3 in severity or classified as SAEs occurred in eight (1.3\%) motavizumab and two $(0.3 \%)$ palivizumab recipients; no level 4 events were reported in either treatment group. A statistically significant higher incidence of skin events considered to be related to study medication was experienced by motavizumab recipients compared with palivizumab recipients, [motavizumab, 10 $(1.6 \%)$; palivizumab, $2(0.3 \%) ; p=0.038]$.There were no cases of anaphylaxis or evidence of respiratory hypersensitivity in either treatment group.

Serious AEs. Although high, the overall incidence of SAEs (motavizumab, 47.2\%; palivizumab, 49.7\%) was consistent with the complexity of the underlying medical conditions of this high-risk infant population with CHD. Because every hospitalization or procedure was captured as the underlying illness, many of the most frequently reported SAEs were listed as the primary cardiac lesion. The most frequently reported SAEs observed in both treatment groups were tetralogy of Fallot (motavizumab, 6.1\%; palivizumab,8.0\%), ventricular septal defect (4.7 versus $5.9 \%$ ), pneumonia (3.2 versus $3.8 \%$ ), and atrioventricular septal defect $(2.1$ versus $3.6 \%)$. SAEs related to treatment were infrequent and comparable between treatment groups [motavizumab, $n=5(0.8 \%)$; palivizumab, $n=6(1.0 \%)]$.

Mortality rates were comparable between the treatment groups [motavizumab, 9 (1.5\%); palivizumab, 10 (1.6\%; Table 2]. No death was deemed related to study drug or RSV or attributable to sudden infant death syndrome. No death was designated by the site investigator as a sudden unexplained death. However, after review by the blinded MedImmune medical monitor, four deaths in each treatment group (motavizumab, 0.6\%; palivizumab, $0.7 \%$ ) were considered to be "sudden unexplained deaths" because they occurred outside the hospital or emergency department without a definitive cause or autopsy performed.

RSV hospitalization and RSV outpatient MALRI. Although this study was not designed or powered as a standalone efficacy study, there was an observed statistically nonsignificant $25 \%$ (RR: $0.75 ; 95 \%$ CI $=0.34-1.59$ ) relative reduction in RSV hospitalization among motavizumab recipients compared with palivizumab recipients. The rates of RSV hospitalization for motavizumab recipients and palivizumab recipients were $1.9 \%(n=12)$ and $2.6 \%(n=16)$, respectively. There was also a statistically nonsignificant $51 \%$ (RR: $0.49 ; 95 \% \mathrm{CI}=0.10-1.99)$ relative reduction in RSV outpatient MALRIs with rates of $1.0 \%(n=3)$ and $1.9 \%(n=6)$ for motavizumab and palivizumab, respectively. RSV hospitalizations among patients with cyanotic CHD were similar for both treatment groups, whereas there were fewer RSV hospitalizations in patients with acyanotic CHD who received motavizumab (Table 3). In both treatment groups, RSV hospitalization rates were numerically higher in the United States than in the EU (motavizumab, 3.9 versus 1.5\%; palivizumab, 5.5 versus $1.9 \%$, respectively). RSV hospitalization rates in the rest of the world were comparable with those found in the EU.
Table 3. Incidence of RSV hospitalization (ITT population)

\begin{tabular}{|c|c|c|c|c|c|}
\hline \multirow[b]{2}{*}{ Population } & \multicolumn{2}{|c|}{ Motavizumab } & \multicolumn{2}{|c|}{ Palivizumab } & \multirow{2}{*}{$\begin{array}{l}\text { Relative risk* } \\
\quad(95 \% \text { CI) }\end{array}$} \\
\hline & $N$ & $n(\%)$ & $N$ & $n(\%)$ & \\
\hline \multicolumn{6}{|l|}{ CHD stratum } \\
\hline Cyanotic $\dagger$ & 346 & $7(2.0)$ & 319 & $7(2.2)$ & $0.93(0.31,2.78)$ \\
\hline Other & 277 & $5(1.8)$ & 293 & $9(3.1)$ & $0.59(0.18,1.76)$ \\
\hline \multicolumn{6}{|l|}{ Geographic region } \\
\hline North America§ & 182 & $6(3.3)$ & 168 & $8(4.8)$ & $0.72(0.23,2.12)$ \\
\hline United States only & 155 & $6(3.9)$ & 146 & $8(5.5)$ & $0.74(0.24,2.19)$ \\
\hline EU\| & 262 & $4(1.5)$ & 261 & $5(1.9)$ & $0.81(0.19,3.21)$ \\
\hline ROWII & 179 & $2(1.1)$ & 183 & $3(1.6)$ & $0.68(0.08,4.56)$ \\
\hline
\end{tabular}

* Relative risk and CI adjusted for the stratification factor of CHD stratum (cyanotic or other).

$\dagger$ Pulmonary atresia with ventricular septal defect, pulmonary atresia with intact ventricular septum, tetralogy of Fallot, single ventricle including hypoplastic left or right heart, tricuspid atresia, double-outlet right ventricle, Ebstein anomaly, or dextrotransposition of the great arteries with/without ventricular septal defect and with/without pulmonary stenosis.

\$Children who did not have one of the designated diagnoses to be included in the cyanotic stratum (above) were classified as "other" anticipating that this group would largely be made up of children with acyanotic CHD lesions.

$\S$ Canada and the United States.

\| Austria, Belgium, Czech Republic, France, Germany, Hungary, Poland, Spain, Sweden, and the United Kingdom.

II Bulgaria, Israel, and Lebanon, Russia.

A similar percentage of subjects with RSV hospitalization in each treatment group required increased supplemental oxygen [motavizumab, 10 (83.3\%); palivizumab, $13(81.3 \%)$ ] were admitted to the intensive care unit [motavizumab, 5 (41.7\%); palivizumab, $6(37.5 \%)]$ and required mechanical ventilation [motavizumab, 2 (16.7\%); palivizumab, 4 (25.0\%)]. The mean (SD) durations in days of RSV hospitalization [motavizumab, 12.7 (14.6); palivizumab 18.9 (27.6)], increased supplemental oxygen use [motavizumab, 8.5 (8.3); palivizumab 19.8 (30.2)], intensive care unit stay [motavizumab, 7.0 (4.4); palivizumab 27.5 (37.8)], and mechanical ventilation [motavizumab, 8.0 (2.8); palivizumab 28.5 (48.4)] were shorter for the motavizumab group compared with the palivizumab group. None of the differences between groups were statistically significant.

Antidrug antibodies and motavizumab serum concentrations. There was a similar rate of ADA observed among patients receiving the corresponding MAb [motavizumab, $n=$ $9(1.5 \%)$; palivizumab, $n=9(1.5 \%)]$. These were all of the IgG class; no IgE was detected in any patient with ADA. The type and frequency of AEs in patients with ADA were generally balanced between treatment groups and were similar to those in patients without detectable ADA. In the motavizumab group, three patients with ADA had a skin event of interest; all three events were transient and none recurred with subsequent study drug doses. No palivizumab patients with ADA experienced a skin event of interest.

A total of $129(20.7 \%)$ motavizumab and $149(24.3 \%)$ palivizumab patients underwent cardiac surgery with cardiopulmonary bypass. Most of these patients (motavizumab, 93.8\% ; palivizumab, $89.9 \%$ ) received $\geq 1$ replacement dose of study drug. A total of 26 patients (motavizumab, 10; palivizumab, 16) received two study drug doses on the same day. Excluding samples obtained after a cardiac surgery requiring 
cardiopulmonary bypass, motavizumab mean trough serum concentrations after doses 2, 3, and 4 were 60.94, 66.59, and $77.87 \mu \mathrm{g} / \mathrm{mL}$, respectively (Table S4, http://links.lww.com/ PDR/A76). Similarly, including those patients who had cardiac surgery requiring cardiopulmonary bypass and who received an additional replacement dose, mean trough serum motavizumab concentrations after doses 2, 3, and 4 were $60.19,65.62$, and $79.01 \mu \mathrm{g} / \mathrm{mL}$, respectively (Table S4, http:// links.lww.com/PDR/A76). There were no significant differences in mean trough serum motavizumab concentrations between patients with cyanotic CHD or acyanotic CHD. Mean trough serum concentrations of motavizumab in children with ADA were lower than in those without detectable ADA $30 \mathrm{~d}$ after dose 1 and significantly lower at subsequent times $(p \leq$ 0.003). Of the patients with ADA in either treatment group, one patient in the palivizumab group was hospitalized for RSV and no patient in either treatment group experienced an RSV outpatient MALRI.

\section{DISCUSSION}

In this study designed to evaluate the safety and tolerance of motavizumab in children aged $\leq 24$ mo with hemodynamically significant CHD, the overall rates of AEs and SAEs in motavizumab recipients were similar to the rates seen in palivizumab recipients. The number of AEs and SAEs reported are indicative of the general poor health of this population, with rates of AEs and SAEs (motavizumab, 93\% and $47 \%$; palivizumab, $92 \%$ and $50 \%$, respectively) seen in this study comparable with those observed in an earlier placebocontrolled study of palivizumab in children with CHD (palivizumab, 96 and 55\%; placebo, 96 and 63\%, respectively) (12). The profile of the most commonly reported AEs is as expected for this population and is consistent with that observed in the previous study by Feltes et al. (12) and in other high-risk children (18). The inclusion criteria for enrollment of children with CHD were the same for this study as for the previous palivizumab cardiac trial (12). A similar distribution of cardiac characteristics among patients enrolled in these two studies reflects comparable study cohorts (Table 1). In the current study, the stratification of cardiac lesions was closely matched between treatment groups (palivizumab versus motavizumab) as were the cardiac characteristics reflecting similar baseline level of cardiac illness (Table S1, http://links. lww.com/PDR/A76).

The mortality rate in the current study was low and similar between the treatment groups and lower than the rates reported in a previously published study of palivizumab in children with hemodynamically significant CHD (12). Given the similarity in underlying heart disease between patients in these two studies, the lower mortality in the current study likely reflects an overall improvement in medical and surgical management of these infants and young children that has occurred over the 9-y interval separating the enrollment periods of the two clinical trials.

As noted by Carbonell-Estrany et al. (18) in a phase 3 efficacy study of premature infants at high risk for serious RSV disease, skin events occurred more frequently in motavi- zumab recipients compared with palivizumab recipients. Similarly, in this study of children with CHD, the incidence of skin events was $\sim 3 \%$ points higher in motavizumab recipients. Also similar was the finding that the majority of the skin events in both groups were nonspecific rashes, and events that were consistent with cutaneous hypersensitivity occurred more often among motavizumab recipients. Skin events occurring within $2 \mathrm{~d}$ of a dose of motavizumab were transient, easily managed, and $\sim 50 \%$ of these subjects were redosed without a recurrence of these events within $2 \mathrm{~d}$ of subsequent study drug doses. Therefore, it is unlikely that these events were immediate hypersensitivity reactions. No events in either group were consistent with anaphylaxis or respiratory hypersensitivity.

Overall, mean \pm SD trough serum concentrations of motavizumab increased with repeated monthly dosing, and the trough serum concentrations were similar for both CHD strata. The average trough serum concentrations of motavizumab and the associated variability in serum concentrations were similar to that observed previously in premature infants (18). The average trough serum concentrations of motavizumab in patients who underwent cardiac surgery with cardiopulmonary bypass and received an extra dose were similar to patients who did not undergo cardiopulmonary bypass, confirming the adequacy of administering an additional dose immediately after surgery. The rate of developing ADA to motavizumab was low overall and similar to that of palivizumab.

The low RSV hospitalization rates observed in both treatment groups were not unexpected because there were two active treatment arms in this study. The control, palivizumab, is very effective in reducing the incidence of RSV hospitalizations. Motavizumab was expected to be at least as effective as palivizumab in the reduction of RSV hospitalizations. In the United States, the RSV hospitalization rate observed in the current study was similar to the rate seen in the palivizumab group in the previous study by Feltes et al. (12), and the US rates in both studies were higher than those noted in other regions. For palivizumab recipients, the RSV hospitalization rate of $5.5 \%$ in the current study is similar to the $5.9 \%$ rate observed in palivizumab recipients in the historical placebo-controlled study in children with CHD (12). The US RSV hospitalization rates in the current study are higher than the rates observed in the EU, a trend mirroring that of the previous study of palivizumab in children with CHD (5.9 versus $3.0 \%$ ) (12).

A limitation of this study in patients with CHD was that it was not designed to statistically confirm efficacy because it was underpowered, and event rates were low. However, efficacy results were consistent with the larger efficacy trial of motavizumab by Carbonell-Estrany et al. in premature infants and children with CLD of prematurity (Fig. 1) (18). In the current study, monthly administration of motavizumab resulted in an observed $25 \%$ relative reduction in the incidence of RSV hospitalization and an observed $51 \%$ relative reduction in the RSV outpatient MALRI rates compared with palivizumab. These results are consistent with the $26 \%$ relative reduction in RSV hospitalizations and $50 \%$ relative reduction in RSV outpatient MALRI reported in infants and 


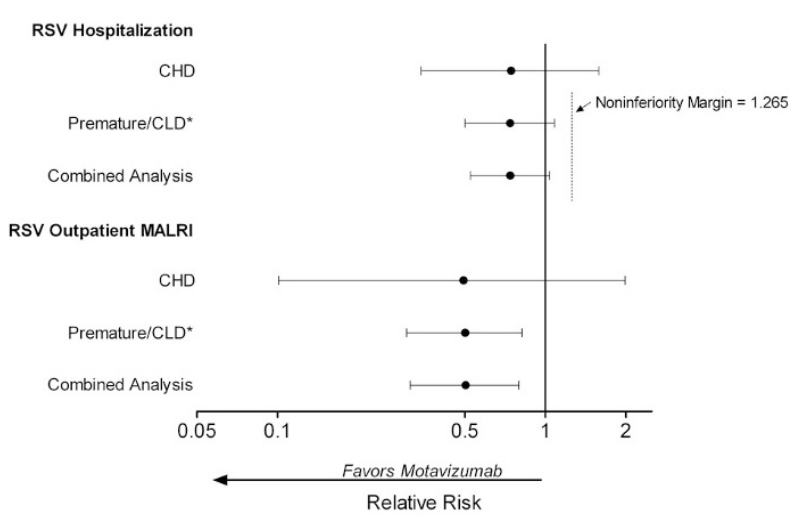

Figure 1. RR of RSV hospitalization or RSV outpatient MALRI in clinical studies. Values below 1.0 represent superiority. Values below 1.265 represent statistical noninferiority. *Premature infants and children with CLD of prematurity. Populations contributing to these analyses: for RSV hospitalization, CHD $(n=1235)$, premature/CLD $(n=6635)$, combined analysis $(n=7870)$; for RSV outpatient MALRI, CHD $(n=614)$, premature/CLD $(n=2410)$, and combined analysis $(n=3024)$.

children at high risk who received motavizumab in the larger efficacy study (18).

In conclusion, in children with hemodynamically significant CHD, motavizumab and palivizumab had similar safety profiles with the one exception of increased skin events in motavizumab recipients. The observed relative reductions in RSV hospitalizations and RSV outpatient MALRI were consistent with those observed in a larger study comparing motavizumab with palivizumab in premature infants.

Acknowledgments. The names of the members of the Motavizumab Cardiac Study Group can be found online as supplemental content (http://links.lww.com/PDR/A76).

We thank the participating children and their parents, the site investigators, study nurses and coordinators, and the clinical testing laboratory staff. In particular, we thank Susan Colbert and Gusiela Torres, who led clinical operations for this study. We also thank Susan E. DeRocco, Ph.D., John E. Fincke, Ph.D., and Gerard P. Johnson, Ph.D., of Complete Healthcare Communications, Inc. (Chadds Ford, PA), for writing and editing assistance funded by MedImmune.

\section{REFERENCES}

1. World Health Organization Acute respiratory infections. Available at: http://www who.int/vaccine_research/diseases/ari/en/index.html. Accessed, January 12, 201
2. Shay DK, Holman RC, Newman RD, Liu LL, Stout JW, Anderson LJ 1999 Bronchiolitis-associated hospitalizations among US children, 1980-1996. JAMA 282:1440-1446

3. Welliver RC 2003 Review of epidemiology and clinical risk factors for severe respiratory syncytial virus (RSV) infection. J Pediatr 143:S112-S117

4. Groothuis JR, Gutierrez KM, Lauer BA 1988 Respiratory syncytial virus infection in children with bronchopulmonary dysplasia. Pediatrics 82:199-203

5. Hall CB, Kopelman AE, Douglas RG Jr, Geiman JM, Meagher MP 1979 Neonatal respiratory syncytial virus infection. N Engl J Med 300:393-396

6. Heilman CA 1990 From the National Institute of Allergy and Infectious Diseases and the World Health Organization. Respiratory syncytial and parainfluenza viruses. J Infect Dis 161:402-406

7. MacDonald NE, Hall CB, Suffin SC, Alexson C, Harris PJ, Manning JA 1982 Respiratory syncytial viral infection in infants with congenital heart disease. $\mathrm{N}$ Engl J Med 307:397-400

8. Johnson S, Oliver C, Prince GA, Hemming VG, Pfarr DS, Wang SC, Dormitzer M, O'Grady J, Koenig S, Tamura JK, Woods R, Bansal G, Couchenour D, Tsao E, Hall WC, Young JF 1997 Development of a humanized monoclonal antibody (MEDI493 ) with potent in vitro and in vivo activity against respiratory syncytial virus. J Infect Dis 176:1215-1224

9. Synagis ${ }^{\circledR}$ (palivizumab). Full Prescribing Information. Available at http://www medimmune.com/pdf/products/synagis_pi.pdf. Accessed April 19, 2011

10. Committee on Infectious Diseases 2009 From the American Academy of Pediatrics: policy statements-modified recommendations for use of palivizumab for prevention of respiratory syncytial virus infections. Pediatrics 124:1694-1701

11. The IMpact-RSV Study Group 1998 Palivizumab, a humanized respiratory syncytial virus monoclonal antibody, reduces hospitalization from respiratory syncytial virus infection in high-risk infants. Pediatrics 102:531-537

12. Feltes TF, Cabalka AK, Meissner HC, Piazza FM, Carlin DA, Top FH Jr, Connor EM, Sondheimer HM 2003 Palivizumab prophylaxis reduces hospitalization due to respiratory syncytial virus in young children with hemodynamically significant congenital heart disease. J Pediatr 143:532-540

13. Mejías A, Chávez-Bueno S, Ríos AM, Aten MF, Raynor B, Peromingo E, Soni P, Olsen KD, Kiener PA, Gómez AM, Jafri HS, Ramilo O 2005 Comparative effects of two neutralizing anti-respiratory syncytial virus (RSV) monoclonal antibodies in the RSV murine model: time versus potency. Antimicrob Agents Chemother 49:4700 4707

14. Wu H, Pfarr DS, Tang Y, An LL, Patel NK, Watkins JD, Huse WD, Kiener PA, Young JF 2005 Ultra-potent antibodies against respiratory syncytial virus: effects of binding kinetics and binding valence on viral neutralization. J Mol Biol 350:126144

15. Wu H, Pfarr DS, Johnson S, Brewah YA, Woods RM, Patel NK, White WI, Young JF, Kiener PA 2007 Development of motavizumab, an ultra-potent antibody for the prevention of respiratory syncytial virus infection in the upper and lower respiratory tract. J Mol Biol 368:652-665

16. Abarca K, Jung E, Fernandez P, Zhao L, Harris B, Connor EM, Losonsky GA 2009 Safety, tolerability, pharmacokinetics, and immunogenicity of motavizumab, a humanized, enhanced-potency monoclonal antibody for the prevention of respiratory syncytial virus infection in at-risk children. Pediatr Infect Dis J 28:267-272

17. Lagos R, Devincenzo JP, Munoz A, Hultquist M, Suzich J, Connor EM, Losonsky GA 2009 Safety and antiviral activity of motavizumab, a respiratory syncytial virus (RSV)-specific humanized monoclonal antibody, when administered to RSVinfected children. Pediatr Infect Dis J 28:835-837

18. Carbonell-Estrany X, Simoes EA, Dagan R, Hall CB, Harris B, Hultquist M, Connor EM, Losonsky GA 2010 Motavizumab for prophylaxis of respiratory syncytial virus in high-risk children: a noninferiority trial. Pediatrics 125:e35-e51

19. Chan IS, Zhang Z 1999 Test-based exact confidence intervals for the difference of two binomial proportions. Biometrics 55:1202-1209

20. Guess HA, Lydick EG, Small RD, Miller LP 1987 Epidemiologic programs for computers and calculators. Exact binomial confidence intervals for the RR in follow-up studies with sparsely stratified incidence density data. Am J Epidemiol 125:340-347 